\title{
Pyrene-based covalent triazine framework towards high-performance sensing and photocatalysis applications
}

\author{
Guang Cheng ${ }^{1 \dagger}$, Kewei Wang ${ }^{2 \dagger}$, Shengyao Wang ${ }^{4 \dagger}$, Liping Guo ${ }^{1}$, Zijian Wang ${ }^{3}$, Jiaxing Jiang ${ }^{3}$, \\ Bien $\operatorname{Tan}^{1}$ and Shangbin Jin
}

\begin{abstract}
Porous organic polymers (POPs) are an emerging class of porous materials, having many promising applications in a variety of areas. Among them, covalent triazine frameworks (CTFs) featuring conjugated and porous structures can be well applied in optoelectronics. To achieve high optoelectronic performance, usually the design and synthesis of CTFs based on appropriate building blocks is critical. Here we report the synthesis of two fluorescent CTFs based on typical fluorescent building blocks, in which CTF-Py constructed from a pyrene (Py) building block was reported for the first time, showing prospective applications in the sensing of nitroaromatics with high sensitivity, and photocatalytic water splitting and carbon dioxide reduction with high performance in comparison with other porous organic materials.
\end{abstract}

Keywords: covalent triazine frameworks, pyrene, fluorescent, water splitting, carbon dioxide reduction

\section{INTRODUCTION}

Porous organic polymers (POPs) which are an emerging class of porous materials derived from organic building blocks have received wide scientific and technical attention $[1,2]$. Various types of structural and functional POPs, such as covalent organic frameworks (COFs) [3-9], conjugated microporous polymers (CMPs) [10-12], hyper-crosslinked polymers (HCPs) [13], polymers of intrinsic microporosity (PIMs) [14,15], and covalent triazine frameworks (CTFs) [16-20], are developed. They have been widely explored in many applications including gas adsorption, catalysis, sensing and optoelectronics $[1,2,20-$ 26]. On the other hand, thin-layered morphology has also extensively attracted enormous attention because of the advantages given by the ultrathin thickness of the materials [27-29]. For example, the thin layers can shorten the migration distances of the excited charges for reducing the recombination probability and the endowed large surface areas would provide abundant sites that are beneficial to chemical sensing and catalysis [30]. The COFs with layered morphologies have been reported as photoluminescent materials and shown good sensing properties [31]. Some CMPs and HCPs with layered morphologies were also reported for photocatalysis $[32,33]$.

CTFs are porous polymers with conjugated structure, high chemical stability and high nitrogen content. The ionothermal synthetic method of CTFs exploited high temperature that went along with partial carbonization, making the CTFs not adaptable for optical applications [16]. After that, a $\mathrm{CF}_{3} \mathrm{SO}_{3} \mathrm{H}$-catalyzed approach was invented to synthesize CTFs, by which fluorescent CTFs could be acquired and their bandgap structures could be retained [20]. The CTFs obtained by this method have exhibited potential optical applications in the sensing of nitroaromatic compounds or photocatalytic water splitting [25]. Recently, a solution synthesis strategy of thinlayered CTFs that were grown in the interface of superacid and solvent was demonstrated for a promising application in field-effect transistor [34]. Although many progresses have been achieved using the above methods,

\footnotetext{
${ }^{1}$ Key Laboratory of Material Chemistry for Energy Conversion and Storage, Ministry of Education-School of Chemistry and Chemical Engineering, Huazhong University of Science and Technology (HUST), Wuhan 430074, China

${ }^{2}$ Department of Chemistry and Chemical Engineering, Shanxi Datong University, Datong 037009, China

${ }^{3}$ School of Materials Science and Engineering, Shaanxi Normal University, Xi'an 710062, China

${ }^{4}$ College of Science, Huazhong Agricultural University, Wuhan 430074, China

${ }^{\dagger}$ These authors contributed equally to this work.

* Corresponding author (email: jinsb@hust.edu.cn)
} 
there are still some disadvantages, such as the partial carbonization or the limitation of the building blocks in the synthesis and applications.

To further overcome these limitations, we have recently shown an effective low-temperature polycondensation reaction to synthesize CTFs at mild conditions, which allows various building blocks to be used [35]. By this route, the CTFs could be designed from versatile building blocks and show high potentials in photocatalysis applications. In order to achieve excellent performance in the applications, it is important to select or design appropriate building blocks in the structures of the polymers. Therefore, many structures have been adopted in the synthesis of CTFs. However, some other meaningful building blocks remain rarely explored in the construction of photofunctional CTFs. For example, fluorescent units like pyrene (Py) and tetraphenylethene (TPE) have been widely explored in the synthesis of porous polymers, such as COFs or CMPs, which can be applied in sensing or other optoelectronic applications [36-39]. TPE block has been reported to synthesize CTF by using the superacid catalysis method $[25,26]$, but the synthesis of TPE-based CTFs using the polycondensation reaction remains undeveloped. Moreover, Py unit has not yet been used in the synthesis of CTFs, although it has already been reported in the synthesis of other useful porous materials for various promising applications [36,37]. Thanks to the highly conjugated structure and fluorescent properties, the synthesis of CTFs based on these building blocks may promisingly result in porous materials with excellent optoelectronic applications.

Along this line, here we report the synthesis of two new CTFs constructed from Py and TPE units by the new polycondensation reaction for the first time. In particular, the pyrene unit was originally used for the synthesis of CTFs, which showed good fluorescence properties and thin-layered morphologies [35]. The as-prepared CTFs have found encouraging performance in chemical sensor for the detection of explosive chemicals with high sensitivity. The CTFs also have great potentials in photocatalysis applications, which exhibit good performance in hydrogen evolution and carbon dioxide reduction thanks to the porous conjugated system and wide visible light absorption. These performances surpass many other reported porous materials.

\section{EXPERIMENTAL SECTION}

\section{Materials}

Methanol, ethanol, 1,4-dioxane, tetrahydrofuran, $\mathrm{N}, \mathrm{N}$-di- methyl formamide (DMF), dimethyl sulfoxide (DMSO), $\mathrm{HCl}$, and anhydrous potassium carbonate were purchased from the National Medicines Corporation Ltd. of China. 1,3,6,8-Tetrabromopyrene and cesium carbonate were purchased from Energy Chemical. 4-Formylphenylboronic acid was purchased from TCI. Tetrakis(triphenylphosphine)palladium was bought from Sigma-Aldrich. Tetra ( $p$-formylphenyl) pyrene (TFPP) and tetra ( $p$-formylbiphenyl)-ethylene (TFBPE) were synthesized according to literature methods [40,41]. Terephthalamidine dihydrochiloride was synthesized according to our previous report [35].

\section{General procedure for the preparation of CTFs}

Synthesis of CTF-Py. A round-bottom flask was charged with TFPP (0.309 g, $0.5 \mathrm{mmol})$, terephthalamidine dihydrochiloride $(0.470 \mathrm{~g}, 2 \mathrm{mmol})$, cesium carbonate $(1.304 \mathrm{~g}, 4 \mathrm{mmol})$ and $10 \mathrm{~mL}$ of DMSO. The mixture was heated at $60^{\circ} \mathrm{C}$ for $12 \mathrm{~h}, 80^{\circ} \mathrm{C}$ for another $12 \mathrm{~h}$, then $100^{\circ} \mathrm{C}$, and finally continuously heated at $120^{\circ} \mathrm{C}$ for three days to obtain an orange solid. The crude product was washed with dilute $\mathrm{HCl}(3 \times 10 \mathrm{~mL})$ to remove the residual cesium carbonate and washed with DMF $(3 \times 10 \mathrm{~mL})$ to remove the unreacted monomer or the oligomer, then washed with ethanol $(3 \times 10 \mathrm{~mL})$ and deionized water $(3 \times 10 \mathrm{~mL})$. The solid was dried by freeze-drying for two days to afford an orange powder $(0.61 \mathrm{~g}, 96 \%$ yield).

Synthesis of CTF-TPE. The procedure was similar to the above except that TFBPE $(0.222 \mathrm{~g}, 0.5 \mathrm{mmol})$ was used instead of TFPP. The final solid was dried by freezedrying for two days to afford an orange powder $(0.46 \mathrm{~g}$, $90 \%$ yield).

\section{Instruments and methods}

The morphologies were measured by using field emission scanning electron microscopy (FE-SEM, FEI Sirion 200 field emission scanning electron microscope). The CTF powders were put on copper studs by using adhesive graphite tape and then coated with carbon before the observation. Nitrogen adsorption/desorption measurements of the CTFs were carried out volumetrically by using a Micromeritics ASAP $2020 \mathrm{M}$ surface area and porosity analyzer (Micromeritics, USA). The surface areas were obtained from the nitrogen adsorption data by Brunauer-Emmett-Teller (BET) or Langmuir equation. Pore size distributions were derived from the isotherms using the nonlocal density functional theory (NLDFT) model based on a slit pore geometry. Thermogravimetric analyses (TGA) of CTFs were carried out using a heating rate of $10^{\circ} \mathrm{C} \mathrm{min}{ }^{-1}$ under a nitrogen atmosphere, with a 
PerkinElmer Diamond TG/DTA instrument. Fourier transform infrared (FT-IR) spectra of CTFs were obtained in the wavenumber range of $4000-400 \mathrm{~cm}^{-1}$ using a Bruker VERTEX 70 FT-IR Spectrometer (Bruker, Germany). UV-visible spectra in the solid-state were characterized by a Shimadzu UV-3600 spectrophotometer. Powder X-ray diffraction (XRD) pattern of CTFs were characterized by a Siemens D5005 diffractometer. Timeresolved fluorescence decay profiles were obtained by Edinburgh instruments FLS-980. Atomic force microscopy (AFM) images of CTFs were observed on a scanning probe microscopy SPM-9700 instrument (Shimadzu Japan). In a typical process for the AFM sample preparation, $2 \mathrm{mg}$ of CTF powders were added into $10-\mathrm{mL}$ centrifuge tubes. Then, $8 \mathrm{~mL}$ of ethanol or isopropanol (IPA) was added into the above tubes as dispersion solvents, and the tubes were sonicated for $10 \mathrm{~h}$, and left undisturbed overnight. Finally, a drop of the dispersed CTF solution was dripped to the mica sheet followed by drying for 15 min under a hot lamp.

\section{Photochemical and electrochemical measurements}

The fluorescence quenching experiments were investigated at room temperature by adding explosive compounds with variable concentrations to the CTF dispersion. For a general procedure, $1 \mathrm{mg}$ of CTFs was dispersed in $40 \mathrm{~mL}$ of DMF with ultrasonication for $30 \mathrm{~min}$, and then different analysts (2,4,6-trinitrophenol (TNP), dinitrotoluene (DNT), nitrobenzene (NB), $p$-nitrotoluene $(p-\mathrm{NT})$, from 0 to $20 \mathrm{ppm})$ were added to the CTFs.

The electrochemical characterizations were conducted in a typical three-electrode cell system by using a CHI60E electrochemical workstation (Chenhua, Shanghai). The working electrodes were prepared on indium-tin-oxide (ITO), which were thoroughly cleaned up by sonication in ethanol for $30 \mathrm{~min}$. The CTF powders $(5 \mathrm{mg})$ were mixed with $0.5 \mathrm{~mL}$ of ethanol and $1 \mathrm{~mL}$ of $1 \%$ Nafion by sonication to get a slurry, which was then spread onto the ITO surface. The uncoated parts of the electrode was separated with epoxy resin, and the exposed area of the electrode was estimated to be $1 \mathrm{~cm}^{2}$. The Pt plate and $\mathrm{Ag} /$ $\mathrm{AgCl}$ electrode $\left(3 \mathrm{~mol} \mathrm{~L}^{-1} \mathrm{KCl}\right)$ were used and worked as the counter electrode and reference electrode, respectively. $0.2 \mathrm{~mol} \mathrm{~L}^{-1} \mathrm{Na}_{2} \mathrm{SO}_{4}$ aqueous solution was used as the electrolyte. The time-correlated photocurrent response measurements were conducted under visible light generated by a $300 \mathrm{~W}$ xenon lamp (Beijing, Perfect light). The electrochemical impedance spectroscopy (EIS) experiments were carried out on the IMP-A.C. Impedance workstation. Mott-Schottky experiments were taken on an IMPE-Impedance Potential workstation, and the potential ranged from -0.3 to $0.8 \mathrm{~V}$. Photocatalytic watersplitting experiments were measured under visible light $(>420 \mathrm{~nm})$ with a $300-\mathrm{W}$ Xe lamp (Perfectlight, PLSSXE300). The CTF samples (50 mg)were dispersed in $100 \mathrm{~mL}$ of aqueous solution with $10 \mathrm{vol} \%$ triethanolamine (TEOA). Pt (3 wt\%) was introduced as a co-catalyst by insitu photodeposition using $\mathrm{H}_{2} \mathrm{PtCl}_{6}$ precursor. The temperature of the solution was maintained at room temperature by using a circulating cooling water system. The hydrogen evolution rates were evaluated by SHIMADZU gas chromatography (GC-2014C) equipped with a thermal conductive detector (TCD) and a $5 \mathrm{~A}$ molecular sieve column. The $\mathrm{CO}_{2}$ photoreduction experiments were performed in a gas-closed system equipped with a gascirculated pump (Makuhari Rikagaku Garasu inc., Japan). The catalyst $(10 \mathrm{mg}), 45 \mathrm{~mL}$ of solution (acetonitrile/ water $=7: 2$ ), $5 \mathrm{~mL}$ of TEOA and the Co (II) bipyridine complexes were mixed in a Pyrex glass reaction cell which was connected to the $\mathrm{CO}_{2}$ reduction system. After evacuation of the catalysis system (no $\mathrm{O}_{2}$ or $\mathrm{N}_{2}$ could be detected by gas chromatography), pure $\mathrm{CO}_{2}$ gas $(\sim 80 \mathrm{kPa})$ was injected into the airtight system. After the adsorption equilibrium, a $300 \mathrm{~W}$ xenon lamp $\left(\sim 100 \mathrm{~mW} \mathrm{~cm}^{-2}\right)$ with a UV-cut filter (L42) was used as the light source $(\lambda>420 \mathrm{~nm})$. The produced $\mathrm{H}_{2}$ and $\mathrm{CO}$ were analyzed by gas chromatographs (GC-2030-BID, Shimadzu Corp., Japan).

\section{RESULTS AND DISCUSSION}

\section{Structural characterization of CTFs}

The CTFs are denoted as CTF-Py and CTF-TPE, respectively (Fig. 1a). The formation of the triazine linkage and network structures were clearly proved by the FT-IR spectra as well as solid-state carbon CP-MAS NMR spectra (Fig. 1b and c). The strong peaks at 1523 and $1355 \mathrm{~cm}^{-1}$ in the FT-IR spectra indicated the formation of a triazine ring in the frameworks.

In the solid-state ${ }^{13} \mathrm{C}$-CP-MAS NMR, the chemical shift at $165.4 \mathrm{ppm}$ was assignable to the carbon signal from triazine rings, confirming the formation of triazine units. The thermogravimetric analysis showed that the CTF-Py had higher thermal stability than CTF-TPE, which was up to $450^{\circ} \mathrm{C}$ (Fig. S1, Supplementary information). When the sample was tested in the oxygen atmosphere at $800^{\circ} \mathrm{C}$, there was little residue after the high-temperature treatment. Fig. S2 shows the contents of the residues are only about 4.2 and 1.9 wt\% for CTF-TPE and CTF-Py, re- 


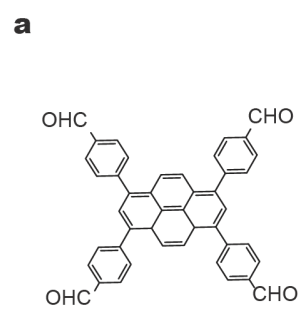

Py monomer

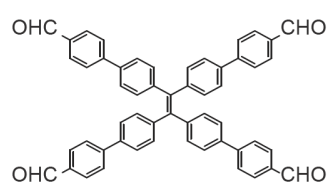

TPE monomer

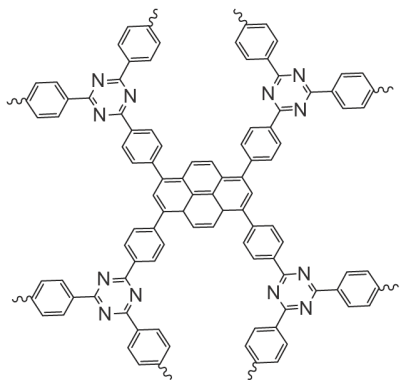

CTF-Py

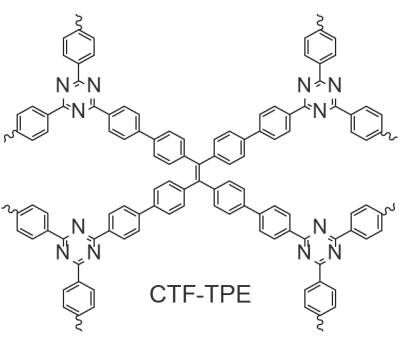

b

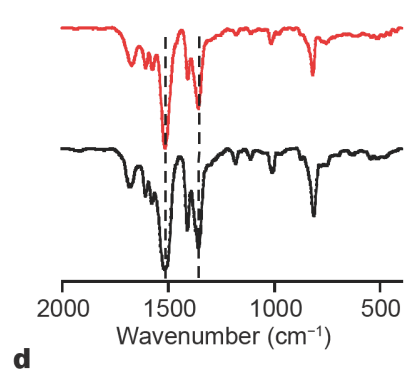

d

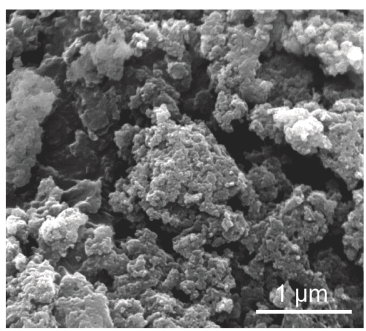

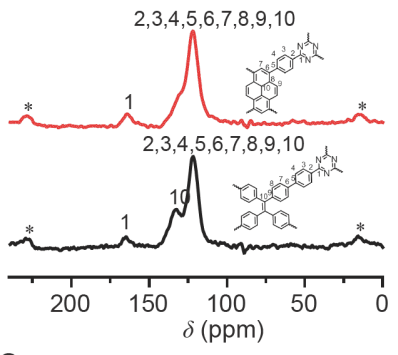

e

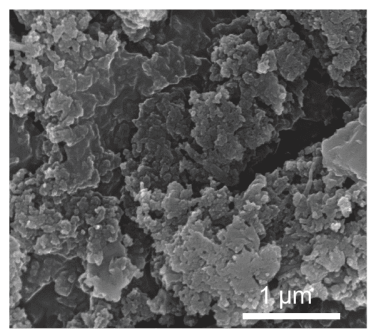

Figure 1 (a) Representative structures of CTF-Py and CTF-TPE and their monomers. (b) FT-IR spectra and (c) CP-MAS- ${ }^{13}$ C-NMR spectra of CTFTPE (black) and CTF-Py (red); FE-SEM images of (d) CTF-TPE and (e) CTF-Py.

spectively, which may be attributed to the residual carbon. Figs S3 and S4 describe the X-ray photoelectron spectroscopy (XPS) results for the CTFs. Signals corresponding to the elements of $\mathrm{C}, \mathrm{N}, \mathrm{O}, \mathrm{Cl}$ and $\mathrm{Cs}$ were observed in the spectrum survey. High-resolution XPS profiles were recorded in the $\mathrm{N} 1 \mathrm{~s}, \mathrm{Cl} 2 \mathrm{p}$, Cs $3 \mathrm{~d}$ regions. The $\mathrm{N}$ 1s spectrum can be deconvoluted into two peaks which are ascribed to pyridinic nitrogen $(399.2 \mathrm{eV}$ for CTF-TPE, $399.0 \mathrm{eV}$ for CTF-Py) and pyrrolic nitrogen (400.5 eV for CTF-TPE, $399.9 \mathrm{eV}$ for CTF-Py). Meanwhile, Fig. S4c and d show the $\mathrm{Cl} 2 \mathrm{p}$ spectrum which can be unambiguously assigned to chloride according to the binding energy derived from the wash reagent [42]. No signal corresponding to Cs $3 \mathrm{~d}$ was detected for both CTFs. Combining the TGA in air and the XPS quantification analysis (Table S1), we may conclude that there was almost no catalyst residual. The elemental analysis of CTFs nearly matched the calculated values (Table S2) derived from the hypothetical fully condensed polymers, with only some small deviations which was normally observed in other microporous materials due to the adsorption capability for water and gases [10].

The morphologies of the resulting CTFs were observed by FE-SEM. CTF-TPE and CTF-Py exhibited particulatelike (Fig. 1d and e) and plate-like morphologies, respectively. The layered morphology was observed by transmission electron microscope (TEM) and high-resolution TEM images (Fig. S5), in which the layer edges were obviously seen from Fig. S5c and d. AFM images further confirmed the sheet-like morphologies of CTFs which may be formed by $\pi-\pi$ stacking of CTF layers. As shown in Figs S6 and S7, CTF-TPE possesses an average layer thickness of around $2 \mathrm{~nm}$ and CTF-Py has an average layer thickness of $4-5 \mathrm{~nm}$. Powder XRD measurements only display broad reflection peaks at around $26^{\circ}$ which can be attributed to the $\pi-\pi$ stacking, revealing the resulting CTFs are disordered (Fig. S8). In principle, the CTFs synthesized in this work should form a three-dimensional (3D) network structures, and they usually do not form 2D layered structures. Unexpectedly, the layered morphologies of CTFs are probably due to the defective $3 \mathrm{D}$ network and $\pi-\pi$ stacking structures.

The nitrogen sorption of the CTFs display the characteristic type IV isotherm curves (Fig. 2a). The BET surface areas of CTF-TPE and CTF-Py were calculated to be 673 and $508 \mathrm{~m}^{2} \mathrm{~g}^{-1}$, respectively. The pore size distributions showed that both CTFs were mainly microporous polymers, with some portions of mesoporous and macroporous structures (Fig. 2b).

\section{Fluorescence properties and sensing application}

Fluorescence spectra shows that CTF-Py and CTF-TPE exhibit yellow fluorescence in the solid-state (Fig. S9). The excitation peak of CTF-Py appears at $469 \mathrm{~nm}$, and its emission peak is found at $544 \mathrm{~nm}$ in the solid-state (Fig. S9a). And the excitation peak of CTF-TPE is observed at $468 \mathrm{~nm}$ and its emission peak emerges at $552 \mathrm{~nm}$ in the solid-state (Fig. S9b). When the CTFs were 

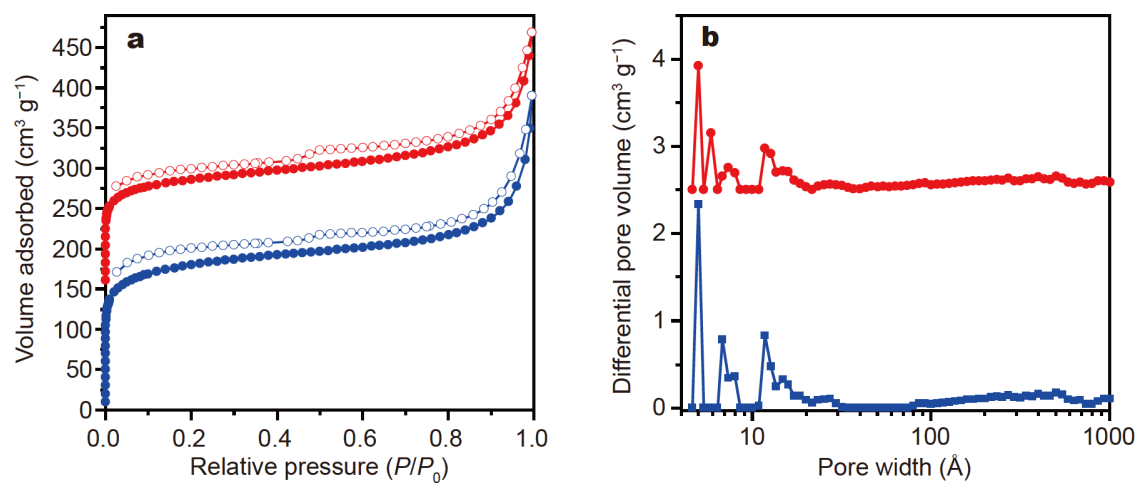

Figure 2 (a) Nitrogen adsorption-desorption isotherms of CTF-Py (red) and CTF-TPE (blue) at 77.3 K, (for clarity, the isotherms of CTF-Py was shifted vertically by $150 \mathrm{~cm}^{3} \mathrm{~g}^{-1}$ ) and (b) pore size distribution calculated using DFT methods (slit pore models, differential pore volumes of CTFHUSTs).

dispersed in DMF, CTF-Py showed an obvious blue shift, in which the excitation peak was found at $396 \mathrm{~nm}$ and the emission peak appeared at $497 \mathrm{~nm}$ (Fig. 3a). However, the CTF-TPE exhibited a much weaker fluorescence in DMF (Fig. S10). The excitation peak of CTF-TPE dispersion was blue-shifted to $436 \mathrm{~nm}$, and the emission peak blueshifted to $535 \mathrm{~nm}[36,37,43,44]$.

After revealing the emission properties of the CTFs, we further investigated their application for sensing some explosive compounds. A series of nitroaromatics with gradient concentrations, i.e., TNP, DNT, NB, $p$-NT, were added to the CTF dispersion in DMF to monitor the emissive response (Fig. $3 \mathrm{~b}$ and Figs S11, S12). The fluorescence is largely quenched when the concentration of TNP was gradually increased in the suspension (Fig. 3b). The fluorescence intensity of CTF-Py is reduced by $81 \%$, while CTF-TPE also shows a $67 \%$ quenching degree when the concentration of TNP is up to $20 \mathrm{ppm}$ (Fig. S13). Therefore, CTF-Py and CTF-TPE are more sensitive to TNP as compared with other nitroaromatics.
The quenching performance of CTF-Py is comparable to other CTFs or COFs in literature reports $[25,44,45]$. For example, the quenching efficiency of CTF-Py for TNP is higher than that of PCTF- 8 which maximally quenches $71 \%$ of the fluorescence emission intensity [25]. It is also higher than some COFs system, such as Py-Azine COF quenching $69 \%$ and $3 \mathrm{D}-\mathrm{Py}-\mathrm{COF}$ quenching $75 \%$ of the fluorescence emission intensity $[45,46]$. The Stern-Volmer curve quenching constant $\left(K_{\mathrm{SV}-\mathrm{TNP}}\right)$ of CTF-Py towards quenching of TNP was estimated to be $5.98 \times 10^{4}\left(\mathrm{~mol} \mathrm{~L}^{-1}\right)^{-1}$ (Fig. S14), which is also comparable to other reported porous materials $[25,47,48]$.

To further understand the quenching mechanism, the fluorescence lifetimes before and after adding TNP were measured. As shown in Fig. S15, the fluorescence lifetime is independent of quenching substance, which suggests a likely static quenching mechanism. As well known, the TNP is a strong protonic acid due to the three nitro groups on the benzene ring. Hence, the CTFs act as bases to be sensitive to the phenolic hydroxyl groups of TNP
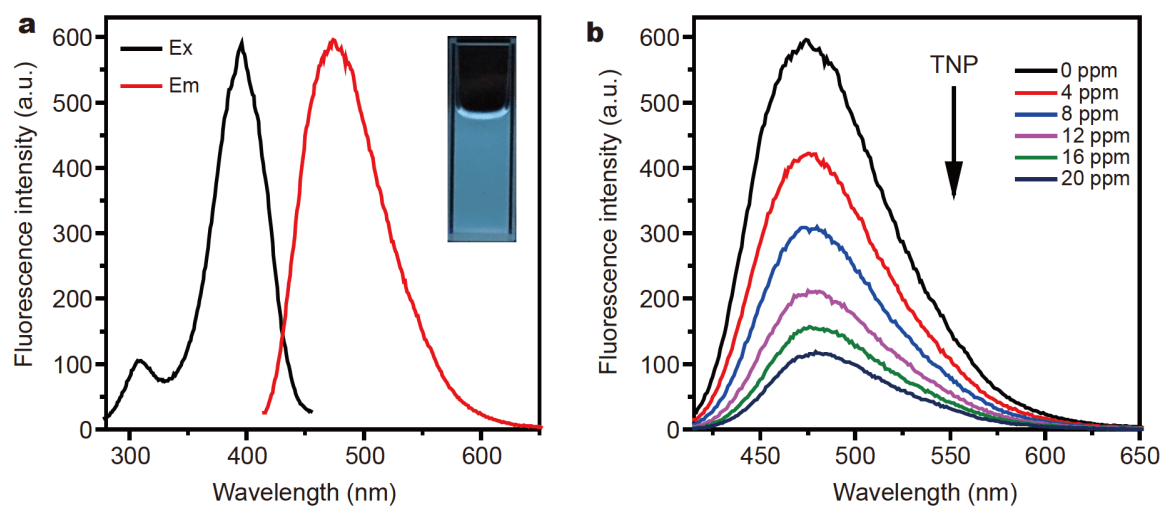

Figure 3 (a) Fluorescence spectra of CTF-Py dispersed in DMF; (b) fluorescence spectra of CTF-Py dispersed in DMF after introduction of different concentrations of TNP. 
molecules and TNP can intensely interact with the triazine ring of CTFs $[25,49]$. Although it is far from the real applications, these results demonstrate the present CTFs are promising candidates for sensing applications.

\section{Photocatalysis applications}

The UV-vis absorption spectra show the CTFs can absorb visible light up to $700 \mathrm{~nm}$ in the solid-state (Fig. 4a). From the Kubelka-Munk-transformed reflectance spectra (Fig. S16), the optical band gap of CTF-Py was estimated to be $2.05 \mathrm{eV}$ and that of CTF-TPE was $2.11 \mathrm{eV}$. Furthermore, the conduction bands and valence bands (CB and $\mathrm{VB}$ ) were calculated based on the electrochemical Mott-Schottky plots (see Fig. S16c and d). The positive slope indicated typical n-type semiconductor behavior for both CTFs. Another meaningful parameter derived from the Mott-Schottky plots is the flat-band potential, which is roughly estimated to be -1.21 and $-1.32 \mathrm{~V}$ versus $\mathrm{Ag} /$ $\mathrm{AgCl}$ for CTF-TPE and CTF-Py, respectively. It is noted that the potential of the CB of CTF-Py is higher than that of CTF-TPE, which may provide a larger dynamic driving force for photocatalysis. Fig. $4 \mathrm{~b}$ shows the time-correlated photocurrent curves, and the periodic currents indicate

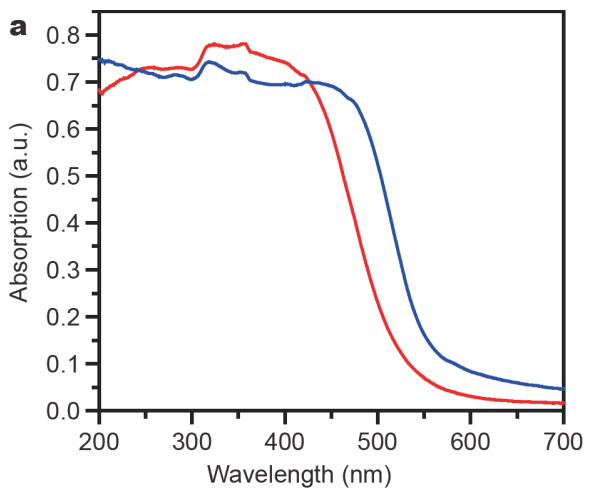

c

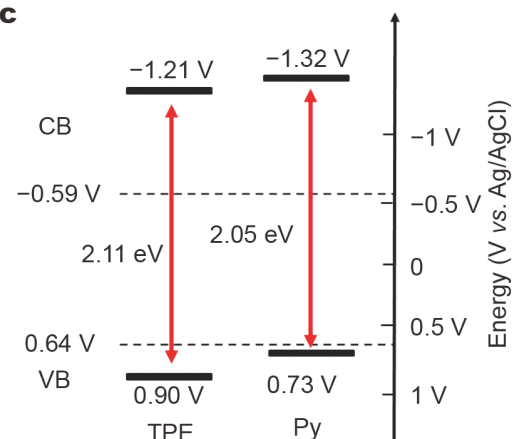

the CTFs are responsive to light irradiation, resulting from the charge separation and transfer. As shown in Fig. $4 c$, the energy level diagrams of the resulting CTFs are described. The conduction bands of the two CTFs are more negative than the $\mathrm{H}^{+} / \mathrm{H}_{2}$ redox potentials, implying the prospective application in the photocatalytic hydrogen evolution. EIS Nyquist plots (Fig. S17) studied the charge transfer rate in the dark. It is expected to have the semicircular Nyquist plots for both CTF-TPE and CTFPy, but a slightly shrunken diameter appears on CTF-Py, indicating the faster charge migration. As shown in Fig. S18, fluorescent decays belong to a double-exponential profile. The fluorescence lifetimes of CTF-TPE are $\tau_{1}=0.72 \mathrm{~ns}(99.04 \%)$ and $\tau_{2}=3.97 \mathrm{~ns}(0.96 \%)$, and the average lifetime is estimated to be $0.75 \mathrm{~ns}$. However, the fluorescence lifetimes of CTF-Py are $\tau_{1}=1.05 \mathrm{~ns}(48.59 \%)$ and $\tau_{2}=4.64 \mathrm{~ns}(51.41 \%)$ and the average lifetime is $2.90 \mathrm{~ns}$ which is longer than the CTF-TPE. The longer fluorescence lifetime means the photogenerated electrons would boost the surface reactions which can promote the photocatalytic activity $[50,51]$.

To show the potentials of the CTFs in the photocatalytic water splitting, the photocatalytic hydrogen
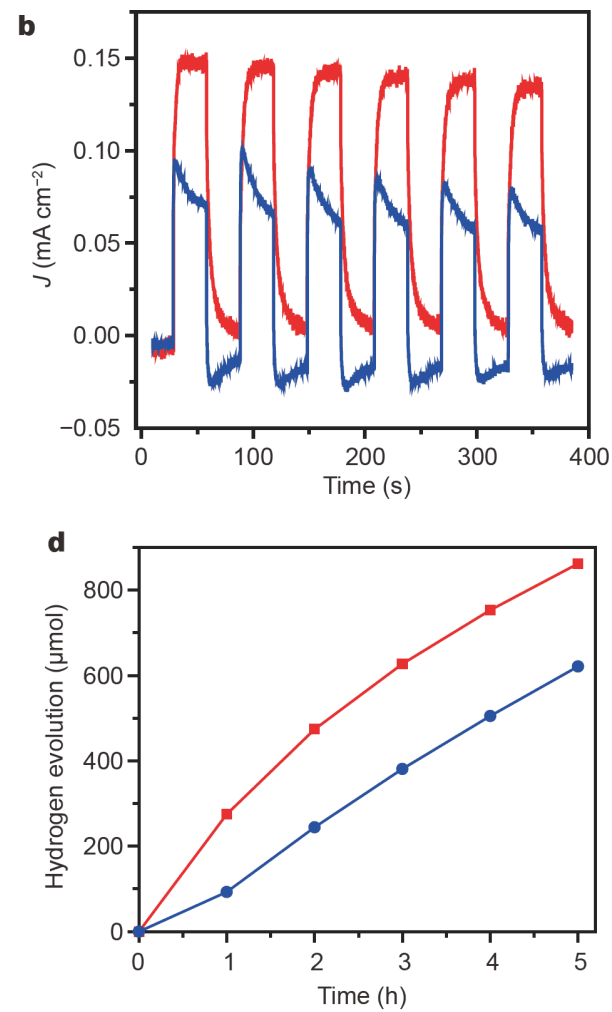

Figure 4 (a) UV-vis spectra of CTF-Py (blue) and CTF-TPE (red); (b) time-correlated photocurrents of CTF-TPE and CTF-Py under visible light irradiation; (c) band structure alignments of CTF-TPE and CTF-Py; (d) time courses of photocatalytic hydrogen evolution of CTF-Py (blue) and CTFTPE (red) under visible light irradiation $(>420 \mathrm{~nm})$. 
evolution was measured in a mixture of $100 \mathrm{~mL}$ TEOA/ $\mathrm{H}_{2} \mathrm{O}(V: V=1: 10)$, in which TEOA worked as the sacrificial electron donor. In addition, $3 \mathrm{wt} \% \mathrm{Pt}$ was loaded on the surface of the materials by an in-situ photoreduction method which played the role of co-catalyst. We can observe that an obvious photoluminescent quenching and a shortening of the photoluminescent lifetime after loading Pt nanoparticles (Figs S19 and S20). It indicated the Schottky junction was formed after loading nanoparticles, resulting in the suppression of the photoexcited charge recombination in CTF-Py and generating a new electron-transfer channel [52]. As revealed in Fig. 4d, both CTFs display high photocatalytic hydrogen evolution performance. CTF-TPE has a hydrogen evolution rate of $125 \mu \mathrm{mol} \mathrm{h}^{-1}\left(2500 \mu \mathrm{mol} \mathrm{h}^{-1} \mathrm{~g}^{-1}\right)$, and CTF-Py possesses a higher rate of $154 \mu \mathrm{mol} \mathrm{h}^{-1}$ $\left(3080 \mu \mathrm{mol} \mathrm{h}^{-1} \mathrm{~g}^{-1}\right)$. The photocatalytic performance of CTF-Py is higher than that of CTF-2 and comparable to other reported CTFs, such as PCTF-8 which showed a photocatalytic activity of $118.5 \mu \mathrm{mol} \mathrm{h}^{-1} \mathrm{~g}^{-1}$, and other porous organic polymers (Table S3) $[8,25,35,53]$. The activity of CTF-Py slightly decreased after several cycles (Fig. S21).

It is noted that longer fluorescence lifetime of CTF-Py than CTF-TPE is better for photocatalysis, while shorter lifetime of Pt@CTF-Py is in favour of photocatalysis. The contradictory trend of the fluorescence lifetime is because the fluorescence is generated via two different processes. In the photocatalysis process, the electrons in CTFs are at first excited and moved to the excited state and then would return to its ground state by fluorescence emission if there is no Pt nanoparticles. After loading with the cocatalyst $\mathrm{Pt}$, the excited electrons are captured and the fluorescence is obviously quenched because of the elec- tron transfer from CTF to Pt nanoparticles. The initial step is actually a charge recombination process, which is generally against photocatalysis if there is no further cocatalyst (Pt nanoparticles). However, if the non-platinized CTFs have longer fluorescence lifetime, the CTF may generate more excited electrons with longer existing time to migrate to Pt nanoparticles and thus it would benefit the photocatalysis process. Therefore, the contradictory trend of the fluorescence lifetime is because of the different mechanisms and both of them are beneficial to the photocatalysis in this work. It is also found that the photocurrent is inconsistent with the photocatalytic performance. This is probably due to the photocatalyisis is mainly influenced by other factors, such as the more negative lowest unoccupied molecular orbital (LUMO) energy levels and the broader light absorption of CTF-Py as compared with CTF-TPE.

Furthermore, photocatalytic carbon dioxide reduction was investigated. The photocatalytic performance of $10 \mathrm{mg}$ CTFs under visible light (with $420 \mathrm{~nm}$ cut-off filter) was measured using $45 \mathrm{~mL}$ of $\mathrm{CH}_{3} \mathrm{CN} / \mathrm{H}_{2} \mathrm{O}$ solvent, $5 \mathrm{~mL}$ of TEOA as the sacrificial agent and $5 \mu \mathrm{mol}$ of $\mathrm{Co}$ (bpy) ${ }_{3} \mathrm{Cl}_{2}$ as a co-catalyst under $\sim 80 \mathrm{kPa}$ of pure $\mathrm{CO}_{2}$ gas over $8 \mathrm{~h}$ (Fig. $5 \mathrm{a}$ ). In the photocatalytic products, both $\mathrm{CO}$ and $\mathrm{H}_{2}$ are found, but the $\mathrm{CO}$ is the major product with a high selectivity. CTF-Py has a high CO evolution rate of $13.73 \mu \mathrm{mol} \mathrm{h}^{-1}\left(1373 \mu \mathrm{mol} \mathrm{h}^{-1} \mathrm{~g}^{-1}\right)$ and a higher CO selectivity of $95.4 \%$ as compared with CTF-TPE (rate of $3.26 \mu \mathrm{mol} \mathrm{h}{ }^{-1}$, selectivity of $85.5 \%$ ). The recycling experiment also shows the CTF-Py has an excellent recyclability over 5 times (Fig. 5b). These values are also comparable to other porous materials (Table S4) [54,55]. The isotope labelling experiment proves the resulting $\mathrm{CO}$ product is originated from $\mathrm{CO}_{2}$ (Figs S22-S24). These
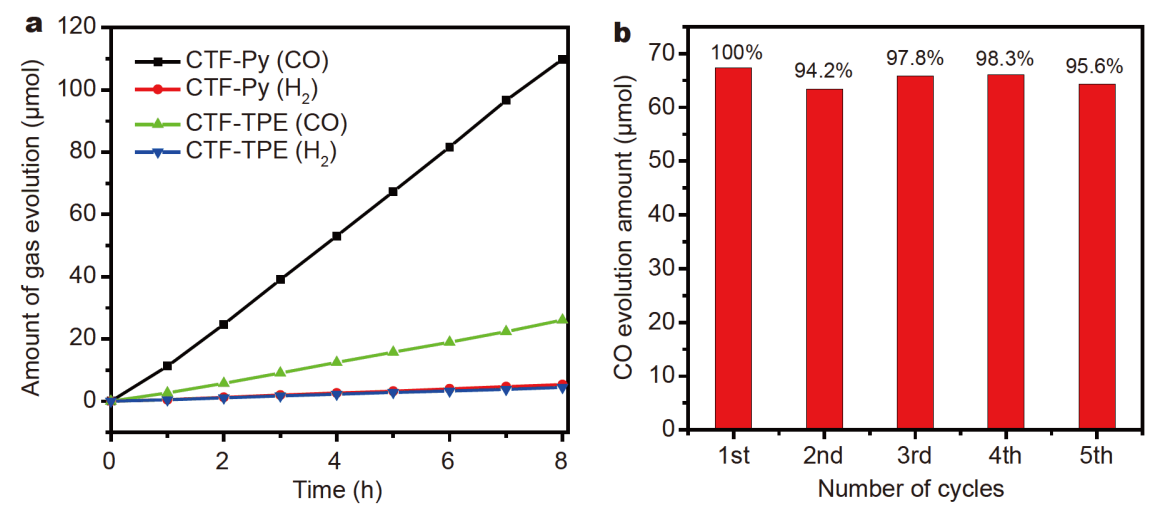

Figure 5 (a) Time courses of the CO evolution for CTFs over 8 h under visible light (using $420 \mathrm{~nm}$ cut-off filter) in $35 \mathrm{~mL} \mathrm{CH} \mathrm{CN}_{3}, 10 \mathrm{~mL} \mathrm{H}_{2} \mathrm{O}, 5 \mathrm{~mL}$ TEOA, and $5 \mu \mathrm{mol} \mathrm{Co(bpy)})_{3} \mathrm{Cl}_{2}$ as a co-catalyst over $10 \mathrm{mg}$ CTFs under $\sim 80 \mathrm{kPa}$ of pure $\mathrm{CO}_{2}$ gas; (b) recyclability of CTF-Py in the photocatalytic $\mathrm{CO}_{2}$ reduction for $5 \mathrm{~h}$ over five cycles. 
results further demonstrate the present CTFs are promising candidates for photocatalysis applications.

\section{CONCLUSIONS}

In summary, we report a new approach to construct fluorescent CTFs based on the polycondensation method. Among them, the resulting layered CTF-Py displays good fluorescence properties, which is potential for sensing application. The broad visible light absorption and layered nanostructures further enable photocatalytic applications in hydrogen evolution and carbon dioxide reduction with high performances. In particular, the photocatalytic carbon dioxide reduction performance is comparable to many other porous materials. Therefore, this work further demonstrates that the polycondensation approach is a useful tool to construct various porous materials for applications.

\section{Received 6 March 2020; accepted 12 April 2020;}

published online 10 July 2020

$1 \mathrm{Wu} \mathrm{D}, \mathrm{Xu} \mathrm{F}$, Sun B, et al. Design and preparation of porous polymers. Chem Rev, 2012, 112: 3959-4015

2 Das S, Heasman P, Ben T, et al. Porous organic materials: Strategic design and structure-function correlation. Chem Rev, 2017, 117: $1515-1563$

3 Cote AP. Porous, crystalline, covalent organic frameworks. Science, 2005, 310: 1166-1170

4 Wan S, Guo J, Kim J, et al. A belt-shaped, blue luminescent, and semiconducting covalent organic framework. Angew Chem Int Ed, 2008, 47: 8826-8830

5 Spitler EL, Dichtel WR. Lewis acid-catalysed formation of twodimensional phthalocyanine covalent organic frameworks. Nat Chem, 2010, 2: 672-677

6 Ding SY, Gao J, Wang Q, et al. Construction of covalent organic framework for catalysis: Pd/COF-LZU1 in Suzuki-Miyaura coupling reaction. J Am Chem Soc, 2011, 133: 19816-19822

7 Kandambeth S, Mallick A, Lukose B, et al. Construction of crystalline $2 \mathrm{D}$ covalent organic frameworks with remarkable chemical (acid/base) stability via a combined reversible and irreversible route. J Am Chem Soc, 2012, 134: 19524-19527

8 Stegbauer L, Schwinghammer K, Lotsch BV. A hydrazone-based covalent organic framework for photocatalytic hydrogen production. Chem Sci, 2014, 5: 2789-2793

9 Pang ZF, Xu SQ, Zhou TY, et al. Construction of covalent organic frameworks bearing three different kinds of pores through the heterostructural mixed linker strategy. J Am Chem Soc, 2016, 138: $4710-4713$

10 Jiang JX, Su F, Trewin A, et al. Conjugated microporous poly (aryleneethynylene) networks. Angew Chem Int Ed, 2007, 46: 8574-8578

11 Cooper AI. Conjugated microporous polymers. Adv Mater, 2009, 21: 1291-1295

12 Chen L, Honsho Y, Seki S, et al. Light-harvesting conjugated microporous polymers: Rapid and highly efficient flow of light energy with a porous polyphenylene framework as antenna. J Am Chem Soc, 2010, 132: 6742-6748
13 Tan L, Tan B. Hypercrosslinked porous polymer materials: Design, synthesis, and applications. Chem Soc Rev, 2017, 46: 3322-3356

14 Budd PM, Ghanem BS, Makhseed S, et al. Polymers of intrinsic microporosity (PIMs): Robust, solution-processable, organic nanoporous materials. Chem Commun, 2004, 230

15 McKeown NB, Budd PM. Polymers of intrinsic microporosity (PIMs): Organic materials for membrane separations, heterogeneous catalysis and hydrogen storage. Chem Soc Rev, 2006, 35: 675-683

16 Kuhn P, Antonietti M, Thomas A. Porous, covalent triazine-based frameworks prepared by ionothermal synthesis. Angew Chem Int Ed, 2008, 47: 3450-3453

17 Kuhn P, Forget A, Su D, et al. From microporous regular frameworks to mesoporous materials with ultrahigh surface area: Dynamic reorganization of porous polymer networks. J Am Chem Soc, 2008, 130: 13333-13337

18 Bojdys MJ, Jeromenok J, Thomas A, et al. Rational extension of the family of layered, covalent, triazine-based frameworks with regular porosity. Adv Mater, 2010, 22: 2202-2205

19 Zhu X, Tian C, Mahurin SM, et al. A superacid-catalyzed synthesis of porous membranes based on triazine frameworks for $\mathrm{CO}_{2}$ separation. J Am Chem Soc, 2012, 134: 10478-10484

20 Ren S, Bojdys MJ, Dawson R, et al. Porous, fluorescent, covalent triazine-based frameworks via room-temperature and microwaveassisted synthesis. Adv Mater, 2012, 24: 2357-2361

$21 \mathrm{Xu} \mathrm{Y}$, Jin S, Xu H, et al. Conjugated microporous polymers: Design, synthesis and application. Chem Soc Rev, 2013, 42: 8012-8031

$22 \mathrm{Xu} \mathrm{Y,} \mathrm{Nagai} \mathrm{A,} \mathrm{Jiang} \mathrm{D.} \mathrm{Core-shell} \mathrm{conjugated} \mathrm{microporous}$ polymers: A new strategy for exploring color-tunable and -controllable light emissions. Chem Commun, 2013, 49: 1591-1593

23 Dalapati S, Jin E, Addicoat M, et al. Highly emissive covalent organic frameworks. J Am Chem Soc, 2016, 138: 5797-5800

24 Dalapati S, Gu C, Jiang D. Luminescent porous polymers based on aggregation-induced mechanism: Design, synthesis and functions. Small, 2016, 12: 6513-6527

25 Bhunia A, Esquivel D, Dey S, et al. A photoluminescent covalent triazine framework: $\mathrm{CO}_{2}$ adsorption, light-driven hydrogen evolution and sensing of nitroaromatics. J Mater Chem A, 2016, 4: 13450-13457

26 Wang X, Zhang C, Zhao Y, et al. Synthetic control and multifunctional properties of fluorescent covalent triazine-based frameworks. Macromol Rapid Commun, 2016, 37: 323-329

27 Dreyer DR, Park S, Bielawski CW, et al. The chemistry of graphene oxide. Chem Soc Rev, 2010, 39: 228-240

28 Huang $\mathrm{X}$, Zeng Z, Zhang $\mathrm{H}$. Metal dichalcogenide nanosheets: Preparation, properties and applications. Chem Soc Rev, 2013, 42: 1934-1946

29 Pakdel A, Bando Y, Golberg D. Nano boron nitride flatland. Chem Soc Rev, 2014, 43: 934-959

30 Ida S, Ishihara T. Recent progress in two-dimensional oxide photocatalysts for water splitting. J Phys Chem Lett, 2014, 5: 2533-2542

31 Das G, Biswal BP, Kandambeth S, et al. Chemical sensing in two dimensional porous covalent organic nanosheets. Chem Sci, 2015, 6: 3931-3939

32 Wang L, Wan Y, Ding Y, et al. Conjugated microporous polymer nanosheets for overall water splitting using visible light. Adv Mater, 2017, 29: 1702428

33 Wang S, Zhang C, Shu Y, et al. Layered microporous polymers by solvent knitting method. Sci Adv, 2017, 3: e1602610

34 Liu J, Zan W, Li K, et al. Solution synthesis of semiconducting twodimensional polymer via trimerization of carbonitrile. J Am Chem Soc, 2017, 139: 11666-11669 
35 Wang K, Yang LM, Wang X, et al. Covalent triazine frameworks via a low-temperature polycondensation approach. Angew Chem Int Ed, 2017, 56: 14149-14153 Jin E, Li J, Geng K, et al. Designed synthesis of stable light-emitting two-dimensional $\mathrm{sp}^{2}$ carbon-conjugated covalent organic frameworks. Nat Commun, 2018, 9: 4143

37 Stegbauer L, Zech S, Savasci G, et al. Tailor-made photoconductive pyrene-based covalent organic frameworks for visible-light driven hydrogen generation. Adv Energy Mater, 2018, 8: 1703278

$38 \mathrm{Xu}$ Y, Mao N, Zhang C, et al. Rational design of donor- $\pi$-acceptor conjugated microporous polymers for photocatalytic hydrogen production. Appl Catal B-Environ, 2018, 228: 1-9

39 Ding H, Li J, Xie G, et al. An AIEgen-based 3D covalent organic framework for white light-emitting diodes. Nat Commun, 2018, 9: 5234

40 Rabbani MG, Sekizkardes AK, El-Kadri OM, et al. Pyrene-directed growth of nanoporous benzimidazole-linked nanofibers and their application to selective $\mathrm{CO}_{2}$ capture and separation. J Mater Chem, 2012, 22: 25409

41 Luo W, Zhu Y, Zhang J, et al. A dynamic covalent imine gel as a luminescent sensor. Chem Commun, 2014, 50: 11942-11945

42 Kishi K, Ikeda S. X-ray photoelectron spectroscopic study of the reaction of evaporated metal films with chlorine gas. J Phys Chem, 1974, 78: 107-112

43 Yuan WZ, Zhao H, Shen XY, et al. Luminogenic polyacetylenes and conjugated polyelectrolytes: Synthesis, hybridization with carbon nanotubes, aggregation-induced emission, superamplification in emission quenching by explosives, and fluorescent assay for protein quantitation. Macromolecules, 2009, 42: 9400-9411

44 Hong Y, Lam JWY, Tang BZ. Aggregation-induced emission. Chem Soc Rev, 2011, 40: 5361-5388

45 Dalapati S, Jin S, Gao J, et al. An azine-linked covalent organic framework. J Am Chem Soc, 2013, 135: 17310-17313

46 Lin G, Ding H, Yuan D, et al. A pyrene-based, fluorescent threedimensional covalent organic framework. J Am Chem Soc, 2016, 138: 3302-3305

47 Shanmugaraju S, Mukherjee PS. $\pi$-Electron rich small molecule sensors for the recognition of nitroaromatics. Chem Commun, 2015, 51: 16014-16032

48 Mallick A, Garai B, Addicoat MA, et al. Solid state organic amine detection in a photochromic porous metal organic framework. Chem Sci, 2015, 6: 1420-1425

49 Wei W, Lu R, Tang S, et al. Highly cross-linked fluorescent poly (cyclotriphosphazene-co-curcumin) microspheres for the selective detection of picric acid in solution phase. J Mater Chem A, 2015, 3: 4604-4611

50 Jun YS, Lee EZ, Wang X, et al. From melamine-cyanuric acid supramolecular aggregates to carbon nitride hollow spheres. Adv Funct Mater, 2013, 23: 3661-3667

51 Meng N, Ren J, Liu Y, et al. Engineering oxygen-containing and amino groups into two-dimensional atomically-thin porous polymeric carbon nitrogen for enhanced photocatalytic hydrogen production. Energy Environ Sci, 2018, 11: 566-571

52 Xiao J-, Shang Q, Xiong Y, et al. Boosting photocatalytic hydrogen production of a metal-organic framework decorated with platinum nanoparticles: The platinum location matters. Angew Chem Int Ed, 2016, 55: 9389-9393

53 Vyas VS, Haase F, Stegbauer L, et al. A tunable azine covalent organic framework platform for visible light-induced hydrogen generation. Nat Commun, 2015, 6: 8508

54 Zhong H, Hong Z, Yang C, et al. A covalent triazine-based frame- work consisting of donor-acceptor dyads for visible-light-driven photocatalytic $\mathrm{CO}_{2}$ reduction. ChemSusChem, 2019, 12: 4493-4499

55 Fu Z, Wang X, Gardner AM, et al. A stable covalent organic framework for photocatalytic carbon dioxide reduction. Chem Sci, 2020, 11: 543-550

Acknowledgements This work was supported by the National Natural Science Foundation of China (21875078 and 21975146).

Author contributions Cheng G and Wang K designed the experiments and synthesized the materials; Cheng $G$ performed the chemical sensor experiments and the data analyses. Wang $S$ conducted the experiment of photocatalytic $\mathrm{CO}_{2}$ reduction; Guo L and Wang $\mathrm{Z}$ performed the experiments of photocatalytic hydrogen evolution; Jiang J and Tan B contributed to the data analyses and discussion. Jin S conceived the project. Jin $\mathrm{S}$ and Cheng $\mathrm{G}$ co-wrote the manuscript.

Conflict of interest The authors declare that they have no conflict of interest.

Supplementary information Supporting data are available in the online version of the paper.

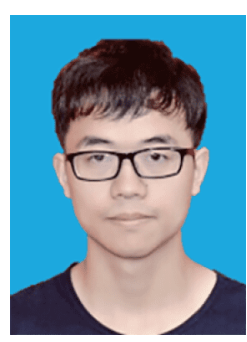

Guang Cheng is a $\mathrm{PhD}$ student at the School of Chemistry and Chemical Engineering, Huazhong University of Science and Technology, China. His research focuses on the preparation of porous organic polymers and their applications.

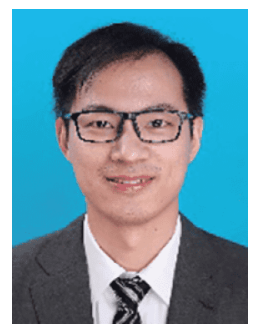

Shangbin Jin is an associate professor at the School of Chemistry and Chemical Engineering, Huazhong University of Science and Technology, China. He received his $\mathrm{PhD}$ degree from the Institute for Molecular Science, the National Institutes of Natural Sciences, Japan, and afterward conducted his postdoc research at the National Institute for Materials Science, Japan. His current research interests mainly focus on the synthesis of covalent triazine frameworks and their photofunctional applications.

\section{面向高性能传感和光催化应用的萠基共价三嗪框架}

程光 ${ }^{1 \dagger}$, 王科伟 ${ }^{2 \dagger}$, 汪圣尧 ${ }^{4 \dagger}$, 郭莉萍 ${ }^{1}$, 王梓鉴 ${ }^{3}$, 蒋加兴 ${ }^{3}$, 谭必恩 ${ }^{1}$, 金尚彬 ${ }^{*}$

摘要 多孔有机聚合物 (POPs) 是一类新兴的多孔材料, 在多个领域 有着广阔的应用前景. 其中, 具有共轭多孔结构的共价三嗪框架 (CTFs) 在光电子学中具有良好的应用前景. 为了获得高性能的光 电材料, 通常基于合适的构建单元设计是构建CTFs至关重要的途 径. 本文报道了两种基于典型苂光构建单元的新型苂光CTF的合 成, 其中CTF-Py是首次由萠(Py)构建单元构建而成, 在高灵敏度硝 基芳烃传感中显示了一定的应用前景, 并在光催化水分解和二氧 化碳还原中展现了良好的催化性能. 